\title{
Acupuncture and transcutaneous electrical nerve stimulation
}

\author{
RONALD MELZACK \\ Ph.D. \\ PATRICK D. WALL \\ M.B., B.Ch., D.M.
}

\begin{abstract}
Department of Psychology, McGill University, Montreal, Quebec, Canada and Department of Anatomy, University College, London, England
\end{abstract}

\section{Introduction}

The study of folk medicine by anthropologists and medical historians has revealed an astonishing array of ingenious methods to relieve pain (Brockbank, 1954). Every culture, it appears, has learned to fight pain with pain: in general, brief, moderate pain tends to abolish severe, prolonged pain. One of the oldest methods is cupping, in which a glass cup is heated up (by coals or flaming alcohol) and then inverted over the painful area and held against it. As the air in the cup cools and contracts, it creates a partial vacuum so that the skin is sucked up into the cup. The procedure produces bruising of the skin with concomitant pain and tenderness. Cupping was practised in ancient Greece and Rome as early as the 4th century B.C., and was also practised in ancient India and China. Over the centuries, the method spread to virtually all parts of the world, and cups of various sizes, shapes and materials have evolved. Cupping has been used-and is still widely practised - for a large variety of ailments, including headaches, backaches, and arthritic pains.

Cauterization is yet another ancient method. Generally, the end of an iron rod was heated until it was red-hot, and was then placed on the painful area, such as the foot in the case of gout, or on the buttock, back or leg in patients with low back pain. Often, however, the cautery was applied to specifically prescribed sites distant from the painful area. The procedure, of course, produced pain and subsequent blistering of the area that was touched by the cautery, but reportedly also led to the relief of chronic pain (Elliott, 1962).

The same effect was achieved by two other procedures: rubbing blistering fluids into the skin, or applying a cone of moxa (made from the leaves of the mugwort plant) to a site on the body, setting the tip of the cone aflame, and allowing it to burn slowly until it approached or reached the skin. Again, the

Correspondence: Dr Ronald Melzack, Department of Psychology, McGill University, 1205 Docteur Penfield Avenue, Montreal, PQ, Canada H3A IBI. procedure produced pain and, while used for all kinds of diseases, was often prescribed specifically for painful conditions (Brockbank, 1954; Elliott, 1962).

There are countless other methods that resemble the ones just described, and which are usually labelled as 'counter-irritation'. It is evident that the one factor common to all of them is that they produce pain to abolish pain. The pain was usually brief and moderate but its effect was to relieve or abolish a much more severe, chronic pain. These methods, of course, did not always work, but they obviously worked well enough to have survived as procedures of folk medicine throughout the world for thousands of years. Do these procedures work better than a placebo? There are no experimental studies, but the evidence from studies of acupuncture suggests that they do.

\section{Acupuncture}

Acupuncture is an ancient Chinese medical procedure that has been in continuous practice for at least 2000 years. It was first described in the western world by the Dutch physician Willem ten Rhyne in 1683. Basically, the procedure involves the insertion of fine needles (made of steel, gold or other metals) through specific points at the skin and then twirling them for some time at a slow rate. The needles may also be left in place for varying periods of time. In recent years, electroacupuncture has been used for painful conditions by electrical stimulation of body tissues through needles hooked up to battery-driven stimulators. The acupuncture needles are inserted into specific sites, adjacent to or distant from the painful area, designated on complex acupuncture charts.

Several kinds of evidence, obtained in western countries as well as in China, reveal the nature of acupuncture's action on pain. The first is the demonstration, in carefully controlled studies, that acupuncture has significantly greater effects on pain than placebo stimulation (Chapman, Wilson and Gehrig, 1976; Anderson, Jamieson and Man, 1974; Stewart, Thomson and Oswald, 1977). However, an impres- 
sive number of studies show that acupuncture stimulation need not be applied at the precise points indicated on acupuncture charts. It is possible, for example, to achieve as much control over dental pain by stimulating an area between the fourth and fifth fingers, which is not designated on acupuncture charts as related to facial pain, as by stimulating the Hoku point between the thumb and index finger which is so designated (Taub et al., 1977). The decreases in pain obtained by stimulation at either site are so large and occur in so many patients that it is unlikely that the pain relief is due to placebo effects. Rather, the results suggest that the site that can be effectively stimulated is not a discrete point but a large area, possibly the whole hand.

That the pain relief produced by acupuncture cannot be attributed simply to a placebo effect is also indicated by the fact that partial analgesia can be produced in animals such as monkeys and mice (Vierck et al., 1974; Pomeranz, Cheng and Law, 1977; Sandrew, Yang and Wang, 1978), and that acupuncture stimulation inhibits or otherwise changes the transmission of pain-evoked nerve impulses at several levels of the central nervous system (Kerr Wilson and Nijensohn, 1978). However, acupuncture needles are not essential to produce these effects. They are also produced by intense electrical stimulation, heat, and a variety of intense sensory inputs (LeBars, Dickenson and Besson, 1979a, b). The effectiveness of all of these forms of stimulation indicates that acupuncture is not a magical procedure, but only one of many ways to produce analgesia by an intense sensory input.

\section{Hyperstimulation analgesia}

After Melzack (1975) had established that brief periods of intense transcutaneous electrical nerve stimulation ('hyperstimulation TENS') were often effective in relieving some forms of chronic pain, Fox and Melzack (1976) carried out a study to compare the relative effectiveness of transcutaneous stimulation and acupuncture on low back pain. The results showed that both forms of stimulation at the same points produce substantial decreases in pain intensity but neither procedure is statistically more effective than the other. Most patients were relieved of pain for several hours, and some for one or more days. Interestingly, an almost identical study was carried out independently in Finland at the same time (Laitinen, 1976) and also found that the two procedures were equally effective in relieving low-back pain.

Our understanding of hyperstimulation analgesia is further enhanced by studies which show that the distribution of acupuncture points is similar to that of trigger points (Travell and Simons, 1983). When acupuncture needles are inserted into sites that reduce pain, they produce a deep, aching feeling when they are twirled manually or electrically stimulated. This is reminiscent of the deep, aching feeling reported by patients when a trigger point is stimulated by the pressure of a finger pushing on it. This similarity led Melzack, Stillwell and Fox (1977) to examine the correlation between trigger points and acupuncture points for pain. The results of their analysis showed that every trigger point reported in the western medical literature has a corresponding acupuncture point. Furthermore, there is a close correspondence $(71 \%)$ between the pain syndromes associated with the two kinds of points. This close correlation suggests that trigger points and acupuncture points related to pain, though discovered independently and labelled differently, represent the same phenomenon and can be explained in terms of similar underlying neural mechanisms.

The relief of pain by brief, intense stimulation of near or distant body sites can be explained in terms of current physiological data. The most plausible explanation (Melzack, 1975) seems to be that the brainstem areas which are known to exert a powerful inhibitory control over transmission in the pain signalling system may be involved. These areas receive inputs from widespread parts of the bodyo and, in turn, project to widespread parts of the spinate cord and brain. The stimulation of particular nerves or tissues by acupuncture needles, transcutaneouselectrical stimulation or any other form of intense stimulation that activates small fibres could bring about an increased input to the reticular formation, which would close the gates to inputs from selected body areas. The cells of the midbrain reticular formation are known to have large receptive fields, and the electrical stimulation of points within the reticular formation can produce analgesia in discrete areas of the body (Soper and Melzack, 1982). It is possible, then, that particular body areas may project especially strongly to some reticular areas, and these, in turn, could 'close the gate' to inputs from particular parts of the body (Melzack and Wall, 1982).

\section{Transcutaneous electrical nerve stimulation}

Transcutaneous electrical nerve stimulation (TENS) (Wall and Sweet, 1967) is the most effective procedure for the sensory modulation of pain. All nerves within about $4 \mathrm{~cm}$ below the surface of the skin can be stimulated by placing electrodes on the skin surface. These include the large nerves in the upper and lower arm, the nerves in the lower leg, and any superficial skin nerves. The electrodes, these days, are usually made of flexible conducting silicone and they make contact with the skin through a 
conducting paste. The electrodes are connected to a pocket-sized, battery-operated stimulator which puts out a continuous series of electrical pulses. The frequency and duration of the pulses vary among different stimulators, but in all of them the strength (amplitude) of the pulses can be varied by the patient himself. The patient raises the strength of stimulation until a comfortable tingling is felt in the area supplied by the nerve which is being stimulated.

This technique has now been extensively used (Woolf, 1984). There is usually a decrease of pain during the stimulation and this is satisfactory for the continuous control of the pain in a substantial percentage of the cases. The most clearcut responses have been obtained when there is skin tenderness associated with nerve damage or disease, or when there are tender muscle points. In patients with causalgia - the most dramatic example of pain associated with localized nerve damage-stimulation central to the area of damage produces a striking decrease in the skin's sensitivity while stimulation peripheral to the damage increases the pain. In postherpetic neuralgia, patients whose main complaint is an unbearable sensitivity of the skin report a satisfactory return of normal sensitivity when the main affected nerves are stimulated (Nathan and Wall, 1974). Since the procedure is so simple and free of side effects, it has come to be used as an initial treatment for many chronic pain syndromes. It is also used in many centres for acute pains by applying the electrodes around the incision scar at the time of surgical operations; it often increases the patient's comfort and decreases the amount of narcotic needed to control postoperative pain. Similarly, the technique is used widely in Sweden during the first stages of childbirth when the mother frequently feels surges of low back pain during uterine contractions.

The mild increase of pain threshold, particularly in cases of skin tenderness, is sufficient to control pain in many patients during the stimulation. Of even more interest to some patients, particularly those with damage to nerves, the relief outlasts a brief period (15-30 $\mathrm{min}$ ) of stimulation by many hours. This is a remarkable phenomenon in which a brief action produces a very prolonged relief.

There is no longer any doubt that TENS is an effective way to treat chronic pain. It is significantly more effective than a placebo machine when stimulation is administered within the painful area, over a related nerve, and even at a distance from the nerve (Thorsteinsson et al., 1977). In a study of joint pain in patients with rheumatoid arthritis, stimulation near the painful joint at low intensity produced significant pain relief in $75 \%$ of patients. When the stimulation intensity was increased, pain relief was obtained by 95\% (Mannheimer, Lund and Carlsson, 1978). Intensity is clearly an important factor, and so is the frequency of the stimulation, although it may depend on the kind of pain. In a study of rheumatoid arthritis (Mannheimer and Carlsson, 1979), high-frequency $(70 \mathrm{~Hz})$ stimulation was more effective than lowfrequency $(3 \mathrm{~Hz})$. Of 20 patients, 18 reported pain relief with $70 \mathrm{~Hz}$, but only 5 with $3 \mathrm{~Hz}$. Furthermore, the average duration of pain relief with $70 \mathrm{~Hz}$ was 18 $\mathrm{hr}$, while for $3 \mathrm{~Hz}$ it was only 4 hours. In contrast, in a study of 123 patients who had pain due primarily to lesions of the nervous system, low-frequency stimulation was better (Eriksson, Sjolund and Nielzen, 1979). High-frequency $(10-100 \mathrm{~Hz})$ stimulation produced significant pain relief in about $35 \%$ of patients, and an additional $20 \%$ were helped by low-frequency $(1-4 \mathrm{~Hz})$ stimulation. The intensity was below painful levels in all cases, but was sufficiently intense to activate deep structures and produce muscle contractions. The good effects persisted in most patients: after 3 months, $55 \%$ of patients continued to use the treatment. After 1 and 2 years, $41 \%$ and $31 \%$ continued to obtain satisfactory relief from the stimulation. An intriguing finding in this study is that the pain relief by low-frequency stimulation was abolished by naloxone (an opioid antagonist), while relief by high-frequency stimulation was not. This, of course, suggests that each type of relief is mediated by different neural mechanisms.

Perhaps the most exciting feature of TENS is that it produces relief in patients who received little or no relief by other methods, including neurosurgical procedures and anaesthetic blocks. In a group of 30 patients with post-herpetic neuralgia, Nathan and Wall (1974) observed that 11 were helped more by TENS than by any other treatment. In nine patients, pain relief outlasted stimulation by 1-2 hr, and two patients were cured. It is not yet possible to state the optimal frequencies or intensities of stimulation for each kind of pain problem, or the percentages of people helped. But it is clear that a high proportion is helped by appropriate stimulation, that TENS is more effective than any other form of treatment for many patients, and that the proportion may become higher when the correct form of stimulation is found for each pain syndrome, and for each patient.

The original reason for introducing the technique (Wall and Sweet, 1967) still appears valid as a partial explanation of its success. Sensory nerve impulses have mixed effects in the central nervous system, producing both excitation and inhibition. A predominant effect is for the large-diameter afferents to raise the threshold of cells which respond to injury signals. As the continuous stimulation is applied, there is a gradual rise of the threshold of spinal cord cells in their ability to respond. In addition, Wall and Gutnick (1974) discovered that electrical stimulation of peripheral nerves, in addition to producing a central inhibition, also may decrease the abnormal 
excitability of the damaged parts of the peripheral nerve.

In summary, recent advances in pain research and therapy have led us away from the traditional neurosurgical approach to severe chronic pain. They have given rise, instead, to several successful procedures for the control of pain by electrical or other forms of sensory modulation of the somatic input.

\section{References}

Anderson, D.G., Jamieson, J.L. \& MAN, S.C. (1974) Analgesic effects of acupuncture on the pain of ice-water: a double-blind study. Canadian Journal of Psychology, 28, 239.

BrockBank, W. (1954) Ancient Therapeutic Arts. Heinemann, London.

Chapman, C.R., Wilson, M.E. \& Gehrig, J.D. (1976) Comparative effects of acupuncture and transcutaneous stimulation on the perception of painful dental stimuli. Pain, 2, 265.

ElliotT, F.A. (1962) Acupuncture and other forms of counterirritation. Transactions and Studies of the College of Physicians of Philadelphia, 30, 81.

ERIKSSON, M.B.E., SJolund, B.H. \& NiElzen, S. (1979) Long-term results of peripheral conditioning stimulation as an analgesic measure in chronic pain. Pain, 6, 335.

Fox, E.J. \& MELZACK, R. (1976) Transcutaneous electrical stimulation and acupuncture: comparison of treatment for low back pain. Pain, 2, 141.

KeRR, F.W.L., Wilson, P.R. \& NiJensohn, D.E. (1978) Acupuncture reduces the trigeminal evoked response in decerebrate cats. Experimental Neurology, 61, 84.

LAITINEN, J. (1976) Acupuncture and transcutaneous electrical stimulation in the treatment of chronic sacrolumbalgia and ischialgia. American Journal of Chinese Medicine, 4, 169.

LeBars, D., Dickenson, A.H. \& Besson, J.-M. (1979a). Diffuse noxius inhibitory controls (DNIC). I. Effects on dorsal horn convergent neruones in the rat. Pain, 6, 283.

LeBars, D., Dickenson, A.H. \& Besson, J.-M. (1979b) Diffuse noxious inhibitory controls (DNIC). II. Lack of effect on nonconvergent neurones, supraspinal involvement and theoretical implications. Pain, 6, 305.

MANnheimer, C. \& CARlSSON, C.A. (1979) The analgesic effect of transcutaneous electrical nerve stimulation (TNS) in patients with rheumatoid arthritis. A comparative study of different pulse patterns. Pain, 6, 329.
MANnheimer, C., LUND, S. \& CARlsson, C.A. (1978) The effect of transcutaneous electrical nerve stimulation (TNS) on joint pain in $\varnothing$

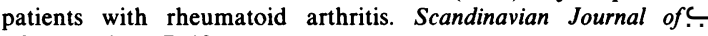
Rheumatology, 7, 13.

MELZACK, R. (1975) Prolonged relief of pain by brief, intense $\overrightarrow{\overline{\vec{S}}}$ transcutaneous somatic stimulation. Pain, 1, 357.

MelzaCK, R., STIllwell, D.M. \& Fox, E.J. (1977) Trigger points and acupuncture points for pain: correlations and implications. Pain, 3, 3.

Melzack, R. \& Wall, P.D. (1982) The Challenge of Pain. Penguin Books, Harmondsworth.

NATHAN, P.W. \& WALL, P.D. (1974) Treatment of post-herpetic neuralgia by prolonged electrical stimulation. British Medical $\vec{O}$ Journal, 3, 645.

PomeranZ, B., Cheng, R. \& LAw, P. (1977) Acupuncture reduces $\vec{\overrightarrow{ }}$ electrophysiological and behavioral responses to noxious stimuli:W pituitary is implicated. Experimental Neurology, 54, 172.

SANDREW, B.B., YANG, R.C.C. \& WANG, S.C. (1978). Electroacu-CO puncture analgesia in monkeys: a behavioral and neurophysiolo- 3 gical assessment. Archives Internationales de Pharmacodynamie et de Therapeutie, 231, 274.

SOPER, W.Y. \& MELZACK, R. (1982) Stimulation-produced analge- $ح$ sia: evidence for somatopic organization in the midbrain. Brain $\bigcirc$ Research, 251, 301.

STEWART, D., THOMSON, J. \& Oswald, D. (1977) Acupuncture analgesia; an experimental investigation. British Medical Journal, $\mathrm{O}$ 1,67 .

TAUb, H.A., BeARD, M.C., EISEnberg, L. \& MCCoRMaCK, R.K. (1977) Studies of acupuncture for operative dentistry. Journal of the American Dental Association, 95, 555.

Thorsteinsson, G., Stonnington, H.H., Stillwell, G.K. \& $\frac{\Phi}{\exists}$ ElVEBACK, L.R. (1977) Transcutaneous electrical stimulation: a double-blind trial of its efficacy for pain. Archives of Physigal Medicine and Rehabilitation, 58, 8.

Travell, J.G. \& Simons, D.G. (1983) Myofascial Pain $\overrightarrow{0}$ Dysfunction: The Trigger Point Manual. Williams and Wilkj色,,+ Baltimore/London.

VIERCK, C.J., LINEBERRY, C.G., LEE, P.K. \& CALDERWOOD, H.W. (1974) Prolonged hypalgesia following 'acupuncture' in monkeys. Life Sciences, 15, 1277.

WALL, P.D. \& GUTNICK, M. (1974) Ongoing activity in peripheral nerves. II. The physiology and pharmcology of impulses originating in a neuroma. Experimental Neurology, 43, 580.

WALL, P.D. \& SWEET, W.H. (1967) Temporary abolition of pain. Science, 155, 108.

WoOLF, C.J. (1984)Transcutaneous and implanted nerve stimulation. In: Textbook of Pain. (Eds. P. D. Wall and R. Melzack), p. 679. Churchill-Livingstone, Edinburgh. 